\title{
Albuminuria indicates the pressure-associated injury of juxtamedullary nephrons and cerebral strain vessels in spontaneously hypertensive stroke-prone rats
}

\author{
Tasuku Nagasawa $^{1}$, Takefumi Mori ${ }^{1}$, Yusuke Ohsaki ${ }^{1}$, Yoshimi Yoneki ${ }^{1}$, Qi Guo ${ }^{1,2}$, Emiko Sato ${ }^{1}$, \\ Ikuko $\mathrm{Oba}^{1}$ and Sadayoshi Ito ${ }^{1}$
}

Albuminuria is an indicator of renal injury and is closely linked with cardiovascular disease (CVD). However, the mechanism by which albumin is excreted in the urine remains unclear. As the juxtamedullary region of the kidney is highly susceptible to pressure increase, juxtamedullary injury is observed from an early phase in hypertensive rat models. Anatomical similarities are observed between the pre-glomerular vessels of the juxtamedullary nephron and the cerebral vasculature. We previously named these 'strain vessels' for their high vascular tone and exposure to higher pressures. The current studies were designed to determine whether albuminuria is the result of juxtamedullary nephron injury, indicating the presence of pressure injury to the strain vessels in spontaneously hypertensive stroke-prone rats (SHR-SP) fed a high-salt diet. Albuminuria was associated with juxtamedullary nephron injury, and the enhanced expression of monocyte chemotactic protein-1 (MCP-1) and tumor growth factor-beta (TGF- $\beta$ ) in 12-week-old SHR-SP rats fed a 4\% high-salt diet from the age of 6 weeks. The wall thickness of the pre-glomerular vessels of the juxtamedullary nephron was also associated with that of the perforating artery of the middle cerebral artery. Reducing the blood pressure with nifedipine reduced the degree of albuminuria and juxtamedullary nephron injury as well as MCP-1 and TGF- $\beta$ expression in the SHR-SP rats fed an $8 \%$ high-salt diet from the age of 9 weeks. Nifedipine inhibited stroke events in these animals until they were 14 weeks old. These results indicate that albuminuria is a result of juxtamedullary nephron injury and a marker of pressure-induced injury of the strain vessels.

Hypertension Research (2012) 35, 1024-1031; doi:10.1038/hr.2012.112; published online 23 August 2012

Keywords: albuminuria; brain; renal juxtamedullary nephron; SHR-SP; strain vessel

\section{INTRODUCTION}

Albuminuria has been increasingly recognized as a well-known predictor for cardiovascular disease (CVD) over the last 10 years. ${ }^{1,2}$ In 1984, Mogensen first reported that microalbuminuria predicted the eventual onset of clinical proteinuria and early mortality in type 2 diabetes. ${ }^{3}$ The presence of albuminuria independently increases the risk for CVD, even in the presence of the known risk factors of hypertension, dyslipidemia and diabetes. ${ }^{4,5}$ Clinical trials have shown that a reduction in albuminuria significantly improved the CVD outcomes in albuminuric subjects (RENAAL, LIFE PREVENT) ${ }^{6-8}$ Surprisingly, albuminuria is recognized as a risk factor not only in diabetic or hypertensive patients but also for the general population. ${ }^{9,10}$ Results from these studies suggest that albuminuria is a sensitive surrogate marker for assessing the risk for CVD. Although extensive investigations have been carried out to determine the mechanism of albuminuria, the conclusions that were drawn from these studies have remained controversial. ${ }^{11}$
In addition, recent clinical studies such as ONTAREGET, TRANSCEND and ACCOMPLISH have failed to demonstrate an association between albuminuria and CVD. ${ }^{12-14}$

It was previously demonstrated that juxtamedullary nephron injury is common in early hypertensive renal injury in rats, as well as in humans. ${ }^{15-17}$ Mori et al. ${ }^{16}$ demonstrated in angiotensin II (Ang II)infused rats that glomerular injury of the juxtamedullary nephron is blood pressure-dependent, while the glomerular integrity of the outer-cortical nephron depends more on Ang II. These results indicate that there is heterogeneity in hypertensive renal injury and that the juxtamedullary nephron may be a source of microalbuminuria in hypertensive subjects. This hypothesis was supported by studies by Ihara et al., ${ }^{18}$ who demonstrated the role of Ang II receptor blockers (ARB) in the reduction of proteinuria in elderly diabetic and hypertensive Otsuka Long-Evans Tokushima Fatty rats. In contrast to hydralazine, olmesartan, an ARB, nearly entirely reduced the levels of proteinuria to a normal baseline level, even for

${ }^{1}$ Division of Nephrology, Endocrinology and Vascular Medicine, Tohoku University Graduate School of Medicine, Sendai, Japan and ${ }^{2}$ Department of Rehabilitation and Sports Medicine, Tianjin Medical University, Tianjin, China

Correspondence: Dr T Mori, Division of Nephrology, Endocrinology and Vascular Medicine, Tohoku University Graduate School of Medicine, 1-1 Seiryocho, Aoba-ku, Sendai 980-8574, Japan. 
blood pressures at which hydralazine had no similar effect. Outer cortical glomerular injury was significantly reduced in the olmesartan group compared with the hydralazine group. However, juxtamedullary glomerular injury was seen in both groups. ${ }^{18}$ Taken together, these results suggest that the juxtamedullary glomeruli were already injured from sustained hypertension, prior to the administration of the ARB, which could be a source of albuminuria.

Wada et al. ${ }^{19}$ performed brain magnetic resonance imaging on 651 elderly subjects and found that the level of albuminuria correlated with the degree of cerebral small vessel disease, independent of the presence of other cerebrovascular risk factors such as diabetes, hypertension and dyslipidemia. We paid particular attention to the anatomical characteristics of the pre-glomerular vessels and the cerebrovascular small vessels, especially the perforating arteries. These vessels are strained under the exposure to high blood pressures, because these vessels branch off directly from the larger, high-velocity arteries. In addition, because the perforating arteries are short and small, and exposed to high pressures, they sustain a large pressure gradient over a short distance. We named this type of vessel a 'strain vessel' and hypothesized that microalbuminuria is a marker of cerebrovascular-renal injury for the strain vessels of these systems. ${ }^{20}$

To test our hypothesis, we determined the association between albuminuria and injury to the strain vessels of spontaneously hypertensive stroke-prone rats (SHR-SP).

\section{MATERIALS AND METHODS}

Protocol 1: Association between albuminuria and cerebrovascularrenal injury

Five-week-old male SHR-SP rats ( $n=16$, Nihon SLC, Hamamatsu, Japan) were fed a $0.5 \% \mathrm{NaCl}$ standard diet for 1 week at baseline and then a high-salt diet ( $4 \% \mathrm{NaCl}$; Nihon Nosan, Yokohama, Japan) for 6 weeks. The animals were given tap water throughout the experimental period. Systolic blood pressures (SBPs) were measured every week in the conscious rats by the tailcuff method (Model MK-2000A; Muromachi, Tokyo, Japan) after reaching 9 weeks of age. Twenty-four-hour urine samples were collected under icecooled conditions in a metabolic cage in the 12-week-old animals, and 24-h urinary albumin excretion (Ualb) was measured by a standard autoanalyzer (Synchron-CX-3; Beckman Coulter Inc., Fullerton, CA, USA). At 12 weeks of age, the rats were euthanized with an intraperitoneal injection of pentobarbital $\left(100 \mathrm{mg} \mathrm{kg}^{-1}\right)$, and the tissues were harvested. All experiments were approved by the Animal Committee of Tohoku University.

\section{Protocol 2: The role of blood pressure in albuminuria and juxtamedullary nephron injury}

To determine whether blood pressure is responsible for the strain vessel injury and the cerebrovascular-renal connection, a number of follow-up protocols were followed. As the timing of albuminuria onset in Protocol 1 was heterogeneous, we decided to shorten the duration of exposure but increase the salt content of the diet, which resulted in a uniform timing of albuminuria onset. Beginning at 9 weeks of age, the SHR-SP rats $(n=16)$ were fed a standard $0.5 \% \mathrm{NaCl}$ diet for 1 week at baseline and then a high-salt diet ( $8 \% \mathrm{NaCl}$; Nihon Nosan) for 2 weeks. The rats were given tap water throughout the experimental period. The animals were randomly divided into two groups. One group served as the control group $(n=8)$, and the other received nifedipine $(n=8)$. Medication administration was started on the day the diets were switched from normal to high salt, and the nifedipine was mixed into the food (Sigma-Aldrich, St Louis, MO, USA, $1.1 \mathrm{~g} \mathrm{~kg}^{-1}$ diet) and administered orally. The rats took in $80-100 \mathrm{mg} \mathrm{kg}^{-1}$ per day of nifedipine on average, as calculated by their food consumption. The SBP and Ualb were measured every week by the same method used for Protocol 1. After a 2-week observation period, the rats were euthanized with an intraperitoneal injection of pentobarbital $\left(100 \mathrm{mg} \mathrm{kg}^{-1}\right)$, and their tissues were harvested.

\section{Histological analysis}

At the end of the Protocol 1 experiment, a saline wash was introduced through the renal artery, and the left kidney was then removed and fixed for $48 \mathrm{~h}(24 \mathrm{~h}$ in $10 \%$ formalin, followed by $24 \mathrm{~h}$ in $100 \%$ ethanol). The tissue was paraffin embedded and sectioned at a thickness of $2 \mu \mathrm{m}$. The renal sections were stained using the periodic acid-Schiff method for renal injury and captured by an optical microscope connected to a CCD video camera (BX51, DP70; Olympus Optical Co., Tokyo, Japan). The glomerular sclerosis index for each sample was determined by grading at least 50 glomeruli per rat, as previously described 21

\section{Immunohistochemical staining}

For alpha-smooth muscle actin ( $\alpha$-SMA), TGF- $\beta$, monocyte chemotactic protein-1 (MCP-1), ED-1 and desmin staining, the residual fixed sample of kidney was retrieved after histological analysis by microwaving the deparaffinized sections in $10 \mathrm{mmoll}^{-1}$ of citrate buffer ( $\mathrm{pH} 7.0$ ) for $5 \mathrm{~min}$. Immunohistochemical analysis was performed using antibodies against $\alpha$-SMA (diluted 1:100; Dako Cytomation, Glostrup, Denmark), TGF- $\beta$ (diluted 1:20, Santa Cruz, CA, USA), MCP-1 (diluted 1:300, Abcam, Cambridge, UK), ED-1 (diluted 1:400, Serotec, Oxford, UK) and desmin (diluted 1:1000, Abcam), and the samples were processed according to previously described methods. ${ }^{22}$ Immunopositive areas were analyzed from 20 randomly captured images with Image J software (NIH software, Ver. 1.37, NIH, Bethesda, MD, USA). The results were reported as the percent of positively stained areas, except for the ED-1 results, which were expressed as the number of immunopositive cells per slide.

At the end of the experiment in Protocol 1, the brains were removed after being perfused, via the left ventricle, with $4 \%$ para-formaldehyde for $5 \mathrm{~min}$. The samples were fixed for $48 \mathrm{~h}$ before they were embedded in paraffin $(24 \mathrm{~h}$ in $10 \%$ formalin, then $6 \mathrm{~h}$ in $70 \%$ ethanol, $6 \mathrm{~h}$ in $80 \%$ ethanol, $6 \mathrm{~h}$ in $90 \%$ ethanol and finally $6 \mathrm{~h}$ in $100 \%$ ethanol). The tissue was embedded in paraffin and sectioned at a thickness of $2 \mu \mathrm{m}$. Following the same method mentioned above, the immunohistochemical analysis was performed using antibodies against $\alpha$-SMA.

Arterioles in the juxtamedullary and/or outer cortical region of the kidney and with an external diameter of 20-30 $\mu \mathrm{m}$ were analyzed. For the brain tissue analysis, any arterioles that were present on the sagittal plane at the level of the optic chiasm were analyzed. Arterioles with a long-axis-to-short-axis ratio $<1.3$ were chosen as suitable for cross-sectional analysis. The medial and luminal cross-sectional areas were quantitatively analyzed and expressed as the percentage of the $\alpha$-SMA-positive arteriolar wall relative to the entire circumferential area of the arteriole, which was determined from the sections that were immunostained for $\alpha$-SMA, using Image J software. With the use of light microscopy and a $\times 400$ magnification, the average wall thickness for each rat was derived as the arithmetic mean of the results for eight arterioles.

\section{RNA extraction and competitive quantitative reverse transcription-} PCR

Total RNA from the renal cortex and outer medulla was extracted using ISOGEN (Nippon Gene, Tokyo, Japan) according to the manufacturer's protocol. Two micrograms of total RNA was used as a template for cDNA synthesis. Complementary DNA was synthesized using SuperScript III FirstStrand Synthesis SuperMix (Invitrogen, Carlsbad, CA, USA) according to the manufacturer's protocol. An aliquot of synthesized cDNA was used as a template for quantitative PCR, using an Applied Biosystems StepOnePlus (Life Technologies Co, Carlsbad, CA, USA). The target cDNAs were amplified by specific primers, using the SYBR Premix Ex Taq solution (Takara Bio, Otsu, Japan) (TGF- $\beta 1$ : forward: $5^{\prime}$-TGCGCCTGCAGAGATTCAAG- $3^{\prime}$, reverse: 5'-AGGTAACGCCAGGAATTGTTGCTA- $3^{\prime}$; osteopontin: forward: $5^{\prime}$-TCCT GCGGCAAGCATTCTC-3' , reverse: 5' -CTGCCAAACTCAGCCACTTTCA-3'; MCP-1: forward: $5^{\prime}$-CTATGCAGGTCTCTGTCACGCTTC- ${ }^{\prime}$, reverse: $5^{\prime}$-CA GCCGACTCATTGGGATCA-3'; glyceraldehyde-3-phosphate dehydrogenase (GAPDH): forward: 5'-GGCACAGTCAAGGCTGAGAATG-3' ${ }^{\prime}$, reverse: 5'-ATG GTGGTGAAGACGCCAGTA- $3^{\prime}$ ). The relative levels of mRNA expression were normalized against the level of GAPDH mRNA expression. 
Protocol 3: The role of blood pressure in cerebrovascular events To further evaluate the effects of blood pressure on the small vessels in the brain, we followed the survival rate of salt-loaded SHR-SP rats for up to 5 weeks. This protocol is the same as Protocol 2, except that the rats were immediately euthanized and their organs harvested if a stroke event (seizure, palsy) was observed. Once strokes were observed in all rats in the control group, all rats in the nifedipine group were euthanized, and their organs were harvested.

\section{Statistical analysis}

The data are presented as the mean \pm s.e.m. Inter-group comparisons of SBP and Ualb measurements taken over the study period were analyzed by repeated-measures ANOVA and the Holm-Sidak post-hoc test. All other data analyses were performed using the Bonferroni/Dunn test for multiple comparisons, following a one-way analysis of variance. All analyses were performed using the raw data. A $P$-value of $<0.05$ was considered statistically significant.

\section{RESULTS}

Protocol 1: Association between albuminuria and cerebrovascularrenal injury

Heterogeneity existed among the rats in their daily Ualb concentrations. Thus, we divided up them according to their Ualb concentrations (cutoff level: Ualb $=5 \mathrm{mg}$ per day), into a high-excretion group (HIGH: Ualb $=21.1 \pm 3.6 \mathrm{mg}$ per day, $n=8$ ) and a low-excretion group (LOW: Ualb $=1.2 \pm 0.6 \mathrm{mg}$ per day, $n=8$ ). We compared the physiological and histological parameters of the two groups, as shown

Table 1 Body weight, kidney weight/body weight, heart weight/body weight, systolic blood pressure and urinary albumin excretion after the 6-weeks study in the Protocol 1

\begin{tabular}{lccccc}
\hline & & $K W / B W$ & $H W / B W$ & $S B P$ & Ualb \\
& $B W(g)$ & $(g / g)$ & $(g / g)$ & $\begin{array}{c}(m m H g) \\
(m g \text { per day })\end{array}$ \\
\hline LOW $(n=8)$ & $244 \pm 3$ & $0.44 \pm 0.01$ & $0.52 \pm 0.02$ & $194 \pm 13$ & $1.2 \pm 0.6$ \\
HIGH $(n=8)$ & $240 \pm 4$ & $0.44 \pm 0.01$ & $0.52 \pm 0.02$ & $205 \pm 23$ & $21.1 \pm 3.6^{*}$
\end{tabular}

Abbreviations: BW, body weight; HIGH, high urinary albumin level (Ualb> $5 \mathrm{mg}$ per day) after the 6 -week study; HW, heart weight; KW, kidney weight; LOW, low urinary albumin level (Ualb $<5 \mathrm{mg}$ per day) after the 6-week study; SBP, systolic blood pressure; Ualb, urinary albumin excretion.

Data are the means \pm s.e. ${ }^{*} P<0.001$ vs. LOW group. below. As shown in Table 1, the mean body weights and the mean heart and kidney masses were not different between the groups. No differences were observed in the 24-h urine volume produced or in the amount of food and water consumed between the groups (data are not shown).

\section{Brain and pre-glomerular arteriolar injury}

We separately analyzed the arterioles of the brain cortex and medulla. As shown in Figures 1a and b, the wall thicknesses of the arterioles in the brain medulla were considerably thicker in the HIGH group than in the LOW group $(82.5 \pm 4.3 \%$ vs. $62.8 \pm 4.2 \%, P<0.05)$. By comparison, the wall thicknesseses of the arterioles in the brain cortex were not significantly different between the two groups $(65.5 \pm 5.6 \%$ vs. $56.0 \pm 3.8 \%, P<0.05)$. We also assessed the arterioles of the renal juxtamedullary region and found that the arteriolar thickness was considerably greater in the HIGH group than in the LOW group $(86 \pm 0.5 \%$ vs. $82 \pm 0.5 \%, P<0.05$, Figure 1c). These results indicate that albuminuria is associated not only with preglomerular arteriolar injury but also with cerebrovascular arteriolar injury.

\section{Glomerular injury}

We hypothesized that albuminuria is a marker of juxtamedullary nephron injury. We separately analyzed the glomeruli of the outer cortical and juxtamedullary nephrons. As shown in Figure 2a, the glomerular sclerosis index scores showed an increased level of glomerulosclerosis in the juxtamedullary glomeruli of the HIGH group compared with the LOW group $(1.4 \pm 0.1$ vs. $0.9 \pm 0.2$, $P<0.05)$. In contrast, the degree of glomerulosclerosis in the outer cortical glomeruli did not differ significantly between the groups $(0.9 \pm 0.2$ vs. $0.6 \pm 0.2$, NS). Overall, the level of glomerulosclerosis was significantly higher in the juxtamedullary glomeruli than in the outer cortical glomeruli of the HIGH group, while no significant differences were observed in the LOW group. To determine whether albuminuria is associated with juxtaglomerular injury, the tissue slides were immunostained with desmin antibodies. As shown in Figure 2f, desmin immunostaining was enhanced in the region of the juxtamedullary nephrons compared with the outer cortical nephrons, indicating that podocyte injury is taking place in the juxtamedullary glomeruli. a
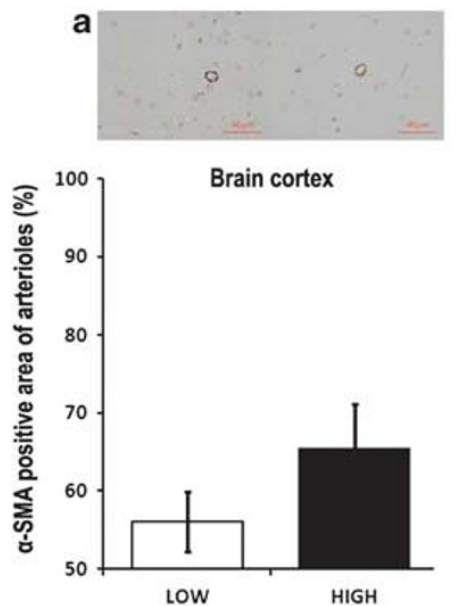

b
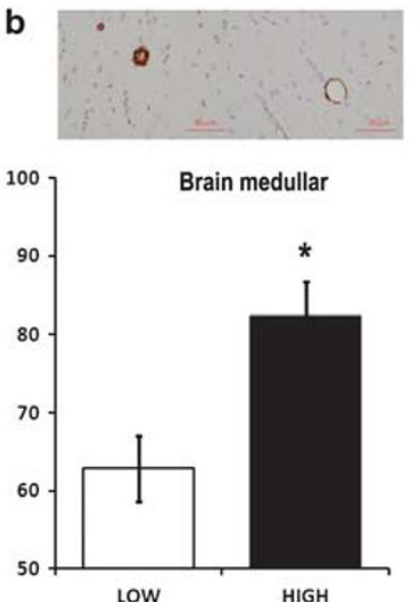

c
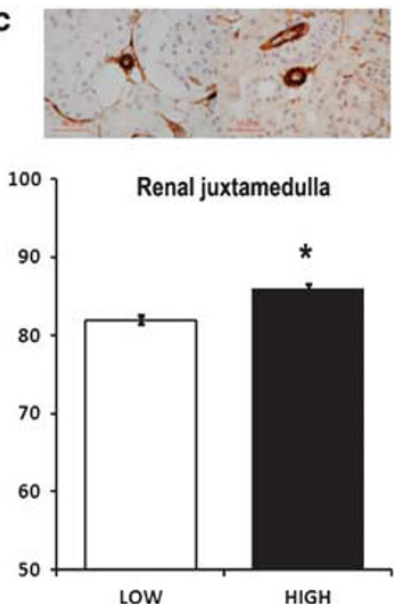

Figure 1 Wall thickness of juxtamedullary preglomerular arteriole and microvessels in brain cortex (a) and medulla (b) determined by $\alpha$-SMA immunostaining. Graphs indicated quantitative representation of positive staining. LOW, low urinary albumin level (Ualb $<5 \mathrm{mg}$ per day; $n=8$ ) after the 6 -weeks study; $\mathrm{HIGH}$, high urinary albumin level (Ualb $>5 \mathrm{mg}$ per day; $n=8$ ) after the 6 -weeks study. Data are the means \pm s.e. ${ }^{*} P<0.05$ vs. LOW group. 
a
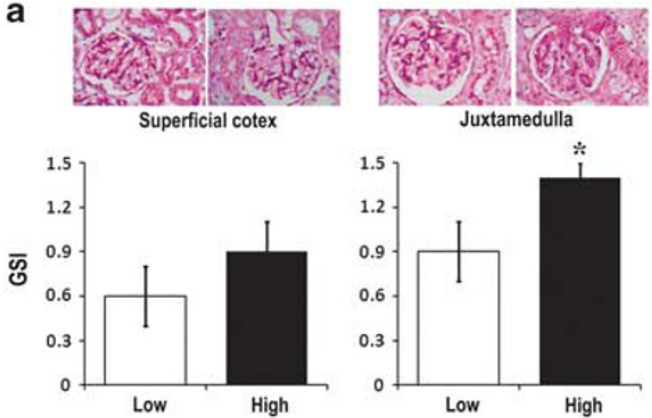

b
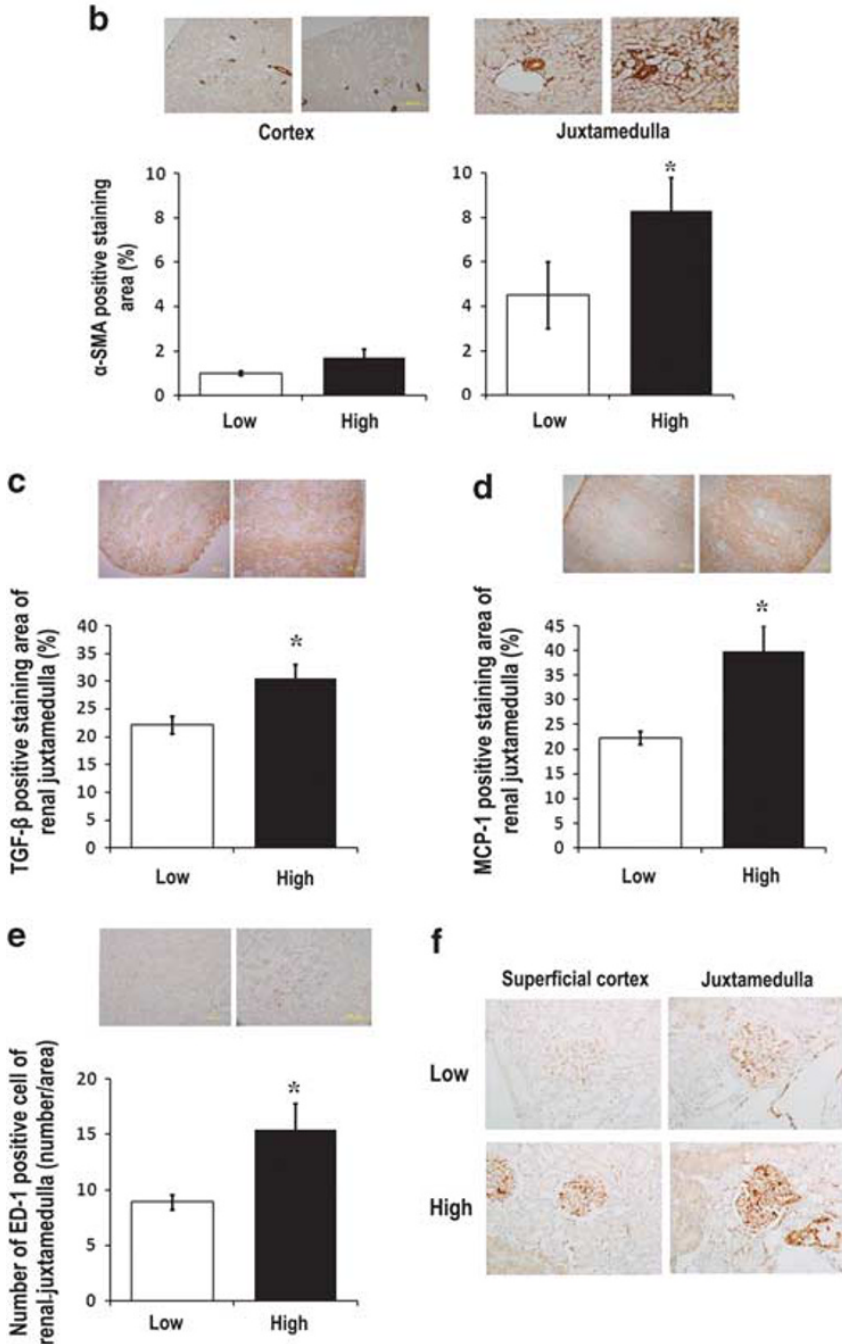

f

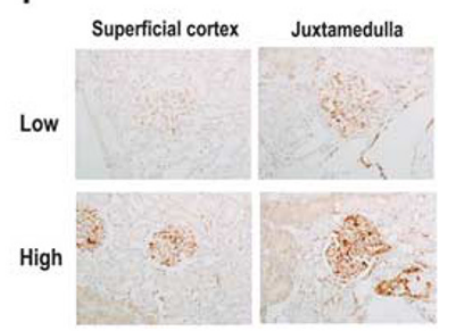

Interstitial and tubular injury

To determine the localization of interstitial injury, the expression of $\alpha$-SMA in the outer renal medulla and cortical region was analyzed. As shown in Figure $2 b$, the expression of $\alpha$-SMA was significantly higher in the outer medullary interstitium of the HIGH group than in the LOW group $(8.3 \pm 1.5 \%$ vs. $4.5 \pm 1.5 \%, P<0.05)$. However, no significant difference existed between the groups in their outer cortical interstitial $\alpha$-SMA expression $(1.7 \pm 0.4 \%$ vs. $1.0 \pm 0.1 \%$, NS), indicating that albuminuria is associated with juxtamedullary nephron injury.

As shown in Figure $2 c$, the percentage of TGF- $\beta$-positive tubules, most prominent in the thick ascending limb and proximal straight tubules of the juxtamedullary region, was significantly higher in the HIGH group than in the LOW group $(30.5 \pm 2.6 \%$ vs. $22.1 \pm 1.6 \%$, $P<0.05$ ). The percentage of MCP-1-positive tubules (Figure 2d), most prominent in the proximal straight tubules of the juxtamedullary region, was also significantly higher in the HIGH group than in the LOW group $(39.8 \pm 5.1 \%$ vs. $22.3 \pm 1.3 \%, P<0.05)$. The number of ED-1-positive cells, infiltrating cells of the interstitium, in the juxtamedullary region (excluding the glomerular tuft, Figure 2e), was significantly greater in the HIGH group compared with the LOW group (15.4 \pm 2.4 per field vs. $8.9 \pm 0.7$ per field, $P<0.05$ ).

Protocol 2: The role of blood pressure in albuminuria and juxtamedullary nephron injury

To determine whether elevated blood pressures are responsible for the juxtamedullary nephron injury in SHR-SP rats, the blood pressures were reduced by administering nifedipine, an L-type $\mathrm{Ca}^{2+}$-channel blocker. Because the timing of albuminuria onset was heterogeneous between the rats in Protocol 1, the animals were fed a high-salt diet over a shortened period time to achieve a uniform increase in albuminuria among the animals. As shown in Figure $3 \mathrm{a}$, the nifedipine-treated rats maintained significantly decreased SBPs
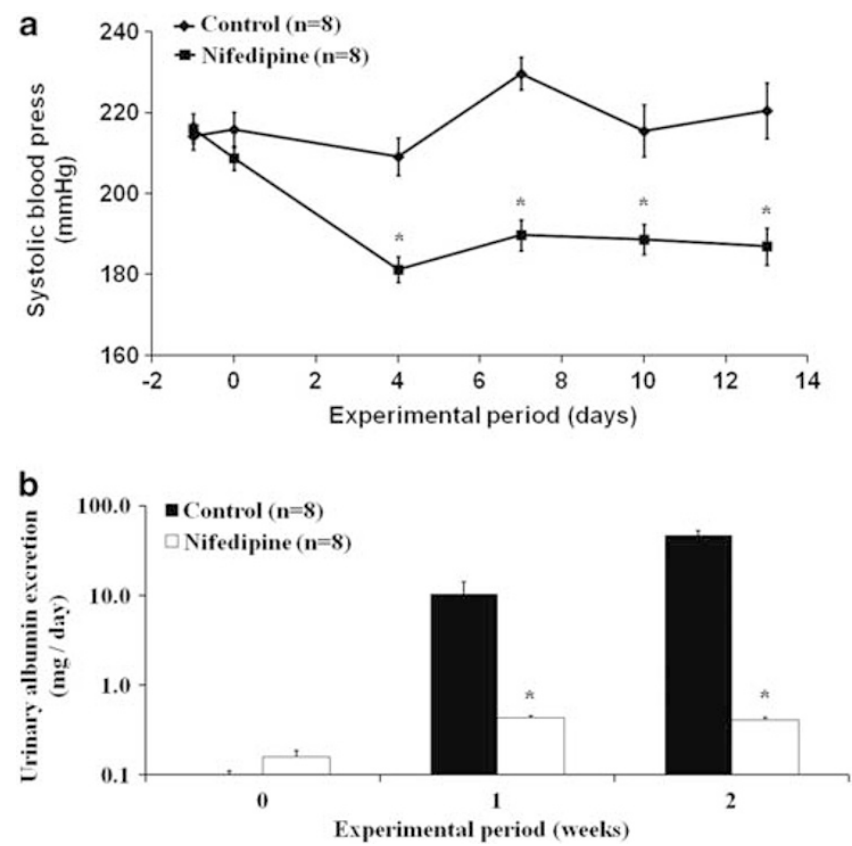

Figure 3 Sequential SBP values (a) and $24 \mathrm{~h}$ Ualb (b) during the 2-week experimental period in Protocol 2. Control, $8 \%$ high-salt-fed SHR-SP; Nifedipine, high-salt-fed SHR-SP treated with nifedipine $\left(80-100 \mathrm{mg} \mathrm{kg}^{-1}\right.$ per day). Data are the means \pm s.e. ${ }^{*} P<0.05$ vs. Control group.
Figure 2 Representative images, and glomerular sclerosis index of superficial cortex and juxtamedulla (a), percentage of $\alpha$-SMA positive staining area of the cortex and juxtamedulla (b), percentage of TGF- $\beta$ (c), MCP-1 (d) positive staining area, and number of ED-1 positive cell (e) of renal juxtamedulla. Representative images of desmin immunostaining of glomerula in renal superficial cortex and juxtamedulla of protocol 1 ( $f$ ). LOW, low urinary albumin level (Ualb $<5 \mathrm{mg}$ per day; $n=8$ ) after the 6-weeks study; HIGH, high urinary albumin level (Ualb > $5 \mathrm{mg}$ per day; $n=8$ ) after the 6-weeks study. Data are the means \pm s.e. ${ }^{*} P<0.05$ vs. LOW group. 
throughout the experimental period $(235 \pm 4$ vs. $191 \pm 2,221 \pm 10$ vs. $166 \pm 6$ and $240 \pm 9$ vs. $190 \pm 3 \mathrm{mmHg}$ on days 6,15 and 20 , respectively, all $P<0.05)$. The mean Ualb for the nifedipine-treated rats was significantly reduced compared with the control rats $(0.4 \pm 0.01$ vs. $10.4 \pm 3.8,0.4 \pm 0.03$ vs. $46.5 \pm 6.1 \mathrm{mg}$ per day at 1 and 2 weeks, $P<0.05$ and $P<0.01$, respectively, Figure $3 \mathrm{~b}$ ). We measured the mRNA levels of inflammatory markers related in the outer renal medulla by the quantitative reverse transcription-PCR method. The nifedipine-treated rats demonstrated significantly decreased mRNA levels of inflammatory markers, cytokines and markers of tubular injury, such as osteopontin, MCP- 1 and TGF- $\beta$, respectively. As shown in Figure 4, the nifedipine group showed reduced levels of mRNA expression for osteopontin, MCP-1, and TGF- $\beta$, all of which co-localized to the tubules in the outer medulla, indicating the involvement of not only the glomeruli but also the tubules of the juxtamedullary nephrons. This occurred because a large portion of the tubules in the outer medulla are from the juxtamedullary nephrons, which was demonstrated in Protocol 1. No major vascular injuries in the brain were observed in either group (data are not shown).

As shown in Figure 5, desmin immunostaining is enhanced in the juxtamedullary nephrons compared with the outer cortical nephrons. This enhancement of desmin immunostaining is attenuated in response to nifedipine treatment, indicating that the reduction of blood pressure is effective in preventing podocyte injury in the juxtamedullary glomeruli.

\section{Pre-glomerular arteriolar injury}

To better delineate the role of the pre-glomerular arterioles, the wall thickness of the juxtamedullary and outer cortical arterioles was determined. As shown in Figure 6, the mean wall thickness of the preglomerular juxtamedullary arterioles was greater than that of the outer cortical arterioles. However, nifedipine reduced the mean juxtamedullary arteriolar wall thickness, indicating that the wall thickness is dependent on blood pressure.

Protocol 3: The role of blood pressure in cerebrovascular events To determine whether blood pressure is responsible for cerebrovascular events, the incidence of stroke events was determined. Figure 7 shows the 35-day, event-free survival curves. The survival analysis showed that the nifedipine group had 35 days of event-free survival, while all of those in the control group suffered an event within the same 35 days (log-rank test, $15.1 ; P<0.01$ ).

\section{DISCUSSION}

The results of this study indicate that albuminuria is associated with glomerular injury, pre-glomerular vascular injury and inflammation of the juxtamedullary nephrons in SHR-SP rats. Lowering the blood pressure in susceptible animals with the L-type $\mathrm{Ca}^{2+}$ antagonist nifedipine inhibited albuminuria and the other indications of glomerular and arteriolar injury. Juxtamedullary nephron injury and albuminuria were associated with injury to the perforating arteries in the cerebral medulla and the incidence of stroke events. To our knowledge, this is the first evidence demonstrating the relationship between albuminuria and juxtamedullary nephron and cerebrovascular injury. We have also demonstrated that the incidence and extent of these injuries was attenuated by using nifedipine to lower the blood pressure, indicating the potential role of blood pressure in explaining the cerebrovascular-renal connection.

\section{Albuminuria is associated with cerebrovascular-renal injury}

The juxtamedullary nephrons extend deep into the renal medulla and branch off either directly from an arcuate artery or from the basal portion of an interlobular artery. Because of the anatomical configuration of the urinary system, the afferent arteriole of a juxtamedullary nephron is exposed to high blood pressures. Glomerular capillary pressure is normally maintained at a constant of approximately $50 \mathrm{mmHg}$ in both the outer-cortical and juxtamedullary glomeruli. ${ }^{20}$ The pre-glomerular vessels, and especially the afferent arterioles, are responsible for regulating the glomerular capillary pressure. The outer-cortical glomeruli can reduce the effective blood pressure from approximately $90 \mathrm{mmHg}$ in the arcuate artery to $50 \mathrm{mmHg}$, over a relatively long distance. By comparison, the juxtamedullary nephron must accomplish this reduction in blood pressure over a much shorter distance. Consequently, the afferent arteriole of the juxtamedullary artery is susceptible to chronic strain from exposure to high pressures and is sensitive to early elevations in blood pressure.

In fact, tissue injury is most obvious in the juxtamedullary region and the outer medulla in spontaneously hypertensive rats (SHR), Dahl salt-sensitive hypertensive rats, renovascular hypertension and

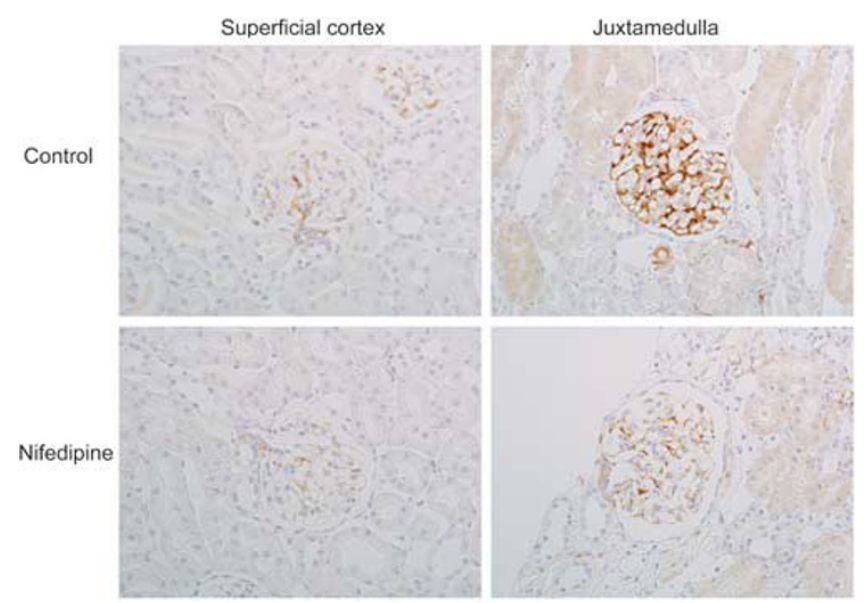

Figure 5 Representative images of desmin immunostaining of glomerula in renal superficial cortex and juxtamedulla of protocol 2 .
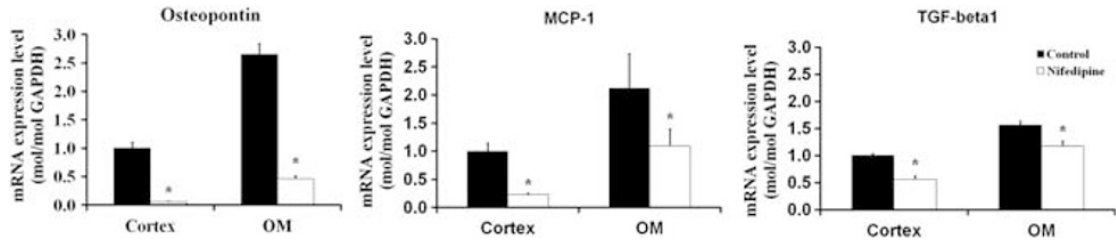

Figure 4 The mRNA levels of osteopontin, MCP-1 and TGF- $\beta 1$ in the renal cortex and outer medullary. Control, $8 \%$ high-salt-fed SHR-SP; Nifedipine, highsalt-fed SHR-SP treated with nifedipine ( $80-100 \mathrm{mg} \mathrm{kg}^{-1}$ per day). Data are the means \pm s.e. $n=5-6$ for each group. ${ }^{*} P<0.05$ vs. Control group. 


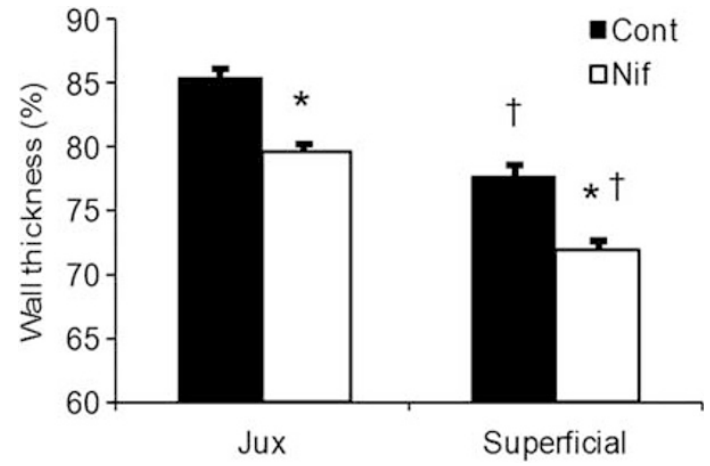

Figure 6 Wall thickness of juxtamedullary and outercortiacal preglomerular arteriole determined by $\alpha$-SMA immunostaining in Protocol 2. Graphs indicated quantitative representation of positive staining. Data are the means \pm s.e. ${ }^{*} P<0.05$ vs. Control (Cont) group, $\dagger P<0.05$ vs. Juxtamedullary glomeruli.

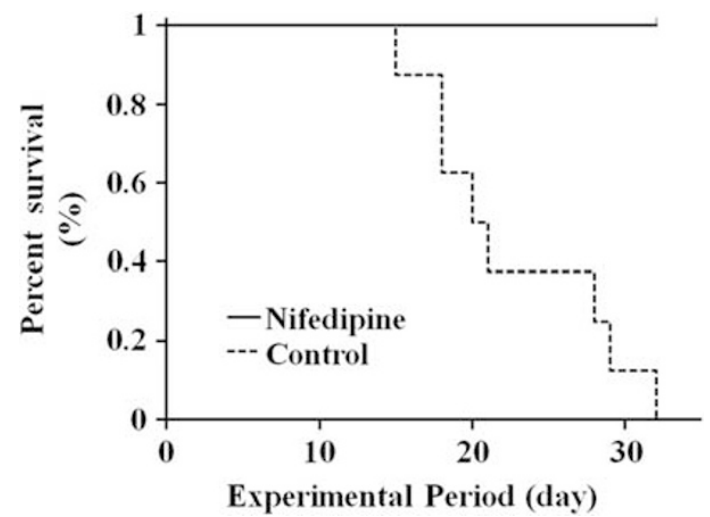

Figure 7 Kaplan-Meier curves for $8 \%$ high-salt-fed SHR-SP with/without nifedipine in the Protocol 3. Control, $8 \%$ high-salt-fed SHR-SP; Nifedipine, high-salt-fed SHR-SP treated with nifedipine $\left(80-100 \mathrm{mg} \mathrm{kg}^{-1}\right.$ per day). $n=8$ in the start of experiment for each group.

Ang II-induced hypertension. ${ }^{15,16,23,24}$ In addition, it has been shown in SHR rats that glomerular lesions first appear predominantly in the juxtamedullary nephrons and then later extend to involve the more superficial nephrons. ${ }^{23}$ This is consistent with the present study's findings of juxtamedullary nephron injury in the absence of any significant injury in the outer cortex. Albuminuria was associated with juxtamedullary nephron injury but not cortical nephron injury, indicating that albumin excretion is predominantly occurring in injured juxtamedullary nephrons.

The wall thickness of the pre-glomerular arteriole of the juxtamedullary nephron is increased when albuminuria is present. In addition, increased wall thickness of the pre-glomerular arteriole is observed in the juxtamedullary nephron without comparable increases in arteriolar wall thickness in the outer cortical nephron. Reducing the blood pressure with nifedipine reduced the wall thickness of these arterioles. Kimura et al. ${ }^{25}$ have demonstrated that the afferent arteriole is constricted and the efferent arteriole is dilated in SHR rats, while glomerular sclerosis is obvious in the juxtamedullary nephron. Our study corroborates these histological findings. Similar to nifedipine in the present study, treatment with captopril or trichlormethiazide plus hydralazine reduced glomerular sclerosis and resulted in the dilatation of the afferent arteriole and constriction of the efferent arteriole. Taken together, the pre- and post-glomerular arterioles have an important role in the development of glomerular sclerosis.

Our previous studies involved the use of a servo-controlled renal perfusion pressure technique in Ang II-infused hypertensive rats and demonstrated that Ang II-dependent renal injury occurred mostly in the outer-cortical glomeruli; by comparison, high blood pressuremediated injury occurred primarily in the juxtamedullary glomeruli. ${ }^{16}$ Interestingly, when this technique was applied to Dahl salt-sensitive rats, which are known to have a lesser capacity to regulate glomerular capillary pressure, they had higher levels of proteinuria after a trial of salt-induced hypertension, and the incidence of glomerular injury depended primarily on the increase in blood pressure, even in the outer cortical glomeruli. These results indicate that the preglomerular vessels are mostly responsible for regulating the glomerular capillary pressure and limiting the damage from continuous exposure to high blood pressures. To determine whether the renin-angiotensin system and/or blood pressure is responsible for the relationship between albuminuria and strain vessel injury, the servo-control technique should be used in future studies with WKY, SHR and SHR-SP rats, both with and without the use of an ARB.

Blood pressure-lowering therapy protects the 'strain vessels' Ihara et al., ${ }^{18}$ using 55-week-old diabetic, hypertensive Otsuka LongEvans Tokushima Fatty rats, demonstrated that using an ARB significantly reduced the levels of proteinuria, compared with hydralazine, in rats who had previously been hypertensive and proteinuric and even though similar reductions in blood pressure were achieved with both the antihypertensive medications. The presence and/or extent of outer cortical glomerular injury was determined by desmin staining, as a marker of podocyte injury, and was reduced by ARB use, while no effect was observed in those animals taking hydralazine. However, the severity of juxtamedullary glomerular injury was not altered with either of the treatments. Why did these antihypertensives fail to prevent juxtamedullary glomerular injury?

When the hydralazine and ARB group of rats were started on the antihypertensive drug, their juxtamedullary glomeruli had already been exposed to high blood pressures, and the animals had already demonstrated significant levels of proteinuria. Concurrently, it was expected that the outer-cortical glomeruli were protected from glomerular hypertension. Because outer cortical glomerular injury is dependent on Ang II, as we observed in a previous study, we hypothesized that ARB administration would protect the outer cortical glomeruli. ${ }^{16}$ These results from our current study support our previous finding that juxtamedullary injury is mostly blood pressure-dependent. Our current study achieved a reduction in the mean SBP by approximately $30 \mathrm{mmHg}$, and this degree of reduction succeeded in preventing stroke and renal juxtamedullary injury in the nifedipine-treated animals. These results strengthen the evidence that elevated blood pressure affects arterioles not only in the brain but also in kidney. In other words, blood pressure-lowering therapy protects 'strain vessels'.

Recent studies have demonstrated that the severity of albuminuria is also dependent on the process of reabsorption in the proximal tubules. ${ }^{26}$ Two basic mechanisms contribute to Ualb. First, there is an uptake of any filtered albumin from the luminal side of the proximal tubule and reabsorption into the surrounding network of peritubular capillaries. ${ }^{11}$ Second, a relatively small amount of albumin is excreted in the urine as a result of peptide metabolism. ${ }^{11}$ In the present study, the renal tubules in the outer medulla, including the proximal straight 
tubules, were injured in the rats with frank albuminuria. This is because the medullary circulation is derived from the efferent arteriole of the juxtamedullary nephron. The relevant mechanisms can be identified by detecting the expression of tubular-injury markers, such as N-acetyl-beta-D-glucosaminidase, beta-2 microglobulin or megalin, which has a key role in the reabsorption of albumin in the proximal tubules. ${ }^{11}$ This was not the focus of the present study, however, and further studies are required. It has been shown that an increase in the renal perfusion pressure increases the renal medullary blood flow, which in turn, increases the renal medullary and cortical interstitial hydrostatic pressures and the peritubular capillary pressure, which together may inhibit $\mathrm{Na}$ reabsorption in the proximal tubules..$^{27,28}$ This mechanism of pressure natriuresis may be impaired when albuminuria is present.

The findings from the present study indicate that albuminuria is the result of juxtamedullary injury. The number of juxtamedullary nephrons is $<10 \%$ the number of cortical nephrons, which supports the findings that albuminuria is limited to a very small amount in the early phase of hypertensive renal injury. However we must acknowledge the possibility that the sclerotic juxtamedullary glomeruli are not the source of albuminuria but rather intact outer cortical glomeruli with increased filtration.

\section{The mechanism of strain vessel injury}

We sought to delineate the mechanism of strain vessel injury, which occurs through a cytokine-mediated inflammatory process. An increase in the renal perfusion pressure has been shown to increase inflammation, which is indicated by the increased number of ED-1positive cells and greater $\alpha$-SMA immunostaining on immunohistochemistry. ${ }^{29,30}$ Although a large portion of its renoprotective effects are attributed to lower blood pressures, nifedipine may function directly as an antioxidant and anti-inflammatory agent. ${ }^{31,32}$ The potentially pleiotropic effects of nifedipine is a limitation of the present study and its mechanism of action in mitigating renal injury requires further study in the future. Because bardoxolone, which is a first-in-class drug being touted as an antioxidant inflammation modulator, has been shown to exert a renoprotective effect, it is possible that nifedipine reduced juxtamedullary injury not only by reducing the blood pressure, but also by exerting an antiinflammatory effect. ${ }^{33}$

In conclusion, the results of this study indicate that albuminuria is a marker for juxtamedullary nephron and pre-glomerular injury in SHR-SP rats. Albuminuria and renal injury were also associated with cerebrovascular injury and the risk for stroke events. The pressureinduced injury of strain vessels may explain the cerebrovascular-renal connection.

\section{ACKNOWLEDGEMENTS}

This study was supported by the Grants-in-Aid for Scientific Research from the Ministry of Education, Culture, Sports, Science and Technology of Japan (Nos. 21390259 and 20590970). In addition, we thank Kiyomi Kisu and Hiroko Ito for their expert technical assistance.

1 Weir MR. Microalbuminuria and cardiovascular disease. Clin J Am Soc Nephrol 2007; 2: 581-590.

2 Karalliedde J, Viberti G. Hypertension and microalbuminuria: risk factors for cardiovascular disease in diabetes. Curr Hypertens Rep 2005; 7: 1-2.

3 Mogensen CE. Microalbuminuria predicts clinical proteinuria and early mortality in maturity-onset diabetes. N Engl J Med 1984; 310: 356-360.

4 Eijkelkamp WB, Zhang Z, Remuzzi G, Parving HH, Cooper ME, Keane WF, Shahinfar S, Gleim GW, Weir MR, Brenner BM, de Zeeuw D. Albuminuria is a target for renoprotective therapy independent from blood pressure in patients with type 2 diabetic nephropathy: post hoc analysis from the Reduction of Endpoints in NIDDM with the Angiotensin II Antagonist Losartan (RENAAL) trial. J Am Soc Nephrol 2007, 18: 1540-1546.

5 Forman JP, Brenner BM. 'Hypertension' and 'microalbuminuria': the bell tolls for thee. Kidney Int 2006; 69: 22-28.

6 de Zeeuw D, Remuzzi G, Parving HH, Keane WF, Zhang Z, Shahinfar S, Snapinn S, Cooper ME, Mitch WE, Brenner BM. Albuminuria, a therapeutic target for cardiovascular protection in type 2 diabetic patients with nephropathy. Circulation 2004; 110 921-927.

7 Ibsen H, Olsen MH, Wachtell K, Borch-Johnsen K, Lindholm LH, Mogensen CE, Dahlof B, Snapinn SM, Wan Y, Lyle PA. Does albuminuria predict cardiovascular outcomes on treatment with losartan versus atenolol in patients with diabetes, hypertension, and left ventricular hypertrophy? The LIFE study. Diabetes Care 2006; 29: 595-600.

8 Hillege HL, Fidler V, Diercks GF, van Gilst WH, de Zeeuw D, van Veldhuisen DJ, Gans RO, Janssen WM, Grobbee DE, de Jong PE. Urinary albumin excretion predicts cardiovascular and noncardiovascular mortality in general population. Circulation 2002; 106: 1777-1782

9 Yuyun MF, Khaw KT, Luben R, Welch A, Bingham S, Day NE, Wareham NJ. Microalbuminuria and stroke in a British population: the European Prospective Investigation into Cancer in Norfolk (EPIC-Norfolk) population study. J Intern Med 2004; 255: 247-256.

10 Hillege HL, Janssen WM, Bak AA, Diercks GF, Grobbee DE, Crijns HJ, Van Gilst WH, De Zeeuw D, De Jong PE. Microalbuminuria is common, also in a nondiabetic, nonhypertensive population, and an independent indicator of cardiovascular risk factors and cardiovascular morbidity. J Intern Med 2001; 249: 519-526.

11 Thomas MC. Pathogenesis and progression of proteinuria. Contrib Nephrol 2011; 170 48-56.

12 Mann JF, Schmieder RE, McQueen M, Dyal L, Schumacher H, Pogue J, Wang X, Maggioni A, Budaj A, Chaithiraphan S, Dickstein K, Keltai M, Metsarinne K, Oto A, Parkhomenko A, Piegas LS, Svendsen TL, Teo KK, Yusuf S. Renal outcomes with telmisartan, ramipril, or both, in people at high vascular risk (the ONTARGET study): a multicentre, randomised, double-blind, controlled trial. Lancet 2008; 372: 547-553.

13 Yusuf S, Teo K, Anderson C, Pogue J, Dyal L, Copland I, Schumacher H, Dagenais G, Sleight P. Effects of the angiotensin-receptor blocker telmisartan on cardiovascular events in high-risk patients intolerant to angiotensin-converting enzyme inhibitors: a randomised controlled trial. Lancet 2008; 372: 1174-1183.

14 Bakris GL, Sarafidis PA, Weir MR, Dahl of B, Pitt B, Jamerson K, Velazquez EJ, StaikosByrne L, Kelly RY, Shi V, Chiang YT, Weber MA. Renal outcomes with different fixeddose combination therapies in patients with hypertension at high risk for cardiovascular events (ACCOMPLISH): a prespecified secondary analysis of a randomised controlled trial. Lancet 2010; 375: 1173-1181.

15 Johnson RJ, Gordon KL, Giachelli C, Kurth T, Skelton MM, Cowley Jr AW. Tubulointerstitial injury and loss of nitric oxide synthases parallel the development of hypertension in the Dahl-SS rat. J Hypertens 2000; 18: 1497-1505.

16 Mori T, Cowley Jr AW. Role of pressure in angiotensin II-induced renal injury: chronic servo-control of renal perfusion pressure in rats. Hypertension 2004. 43: 752-759.

17 Bohle A, Biwer E, Christensen JA. Hyperperfusion injury of the human kidney in different glomerular diseases. Am J Nephrol 1988; 8: 179-186.

18 Ihara G, Kiyomoto H, Kobori H, Nagai Y, Ohashi N, Hitomi H, Nakano D, Pelisch N, Hara T, Mori T, Ito S, Kohno M, Nishiyama A. Regression of superficial glomerular podocyte injury in type 2 diabetic rats with overt albuminuria: effect of angiotensin II blockade. J Hypertens 2010; 28: 2289-2298.

19 Wada M, Nagasawa H, Kurita K, Koyama S, Arawaka S, Kawanami T, Tajima K, Daimon M, Kato T. Microalbuminuria is a risk factor for cerebral small vessel disease in community-based elderly subjects. J Neurol Sci 2007; 255: 27-34.

20 Ito S, Nagasawa T, Abe M, Mori T. Strain vessel hypothesis: a viewpoint for linkage of albuminuria and cerebro-cardiovascular risk. Hypertens Res 2009; 32: 115-121.

21 Tufescu A, Kanazawa M, Ishida A, Lu H, Sasaki Y, Ootaka T, Sato T, Kohzuki M. Combination of exercise and losartan enhances renoprotective and peripheral effects in spontaneously type 2 diabetes mellitus rats with nephropathy. J Hypertens 2008; 26: 312-321.

22 Guo Q, Mori T, Jiang Y, Hu C, Osaki Y, Yoneki Y, Sun Y, Hosoya T, Kawamata A, Ogawa S, Nakayama M, Miyata T, Ito S. Methylglyoxal contributes to the development of insulin resistance and salt sensitivity in Sprague-Dawley rats. J Hypertens 2009; 27 1664-1671.

23 Iversen BM, Amann K, Kvam FI, Wang X, Ofstad J. Increased glomerular capillary pressure and size mediate glomerulosclerosis in SHR juxtamedullary cortex. Am Physiol 1998; 274: F365-F373.

24 Eng E, Veniant M, Floege J, Fingerle J, Alpers CE, Menard J, Clozel JP, Johnson RJ. Renal proliferative and phenotypic changes in rats with two-kidney, one-clip Goldblatt hypertension. Am J Hypertens 1994; 7: 177-185.

25 Kimura K, Tojo A, Matsuoka H, Sugimoto T. Renal arteriolar diameters in spontaneously hypertensive rats. Vascular cast study. Hypertension 1991; 18: 101-110.

26 Vallon $\mathrm{V}$. The proximal tubule in the pathophysiology of the diabetic kidney. Am J Physiol Regul Integr Comp Physiol 2011; 300: R1009-R1022.

27 Jin C, Hu C, Polichnowski A, Mori T, Skelton M, Ito S, Cowley Jr AW. Effects of renal perfusion pressure on renal medullary hydrogen peroxide and nitric oxide production. Hypertension 2009; 53: 1048-1053.

28 Roman RJ. Pressure diuresis mechanism in the control of renal function and arteria pressure. Fed Proc 1986; 45: 2878-2884.

29 Greene AS, Yu ZY, Roman RJ, Cowley Jr AW. Role of blood volume expansion in Dahl rat model of hypertension. Am J Physiol 1990; 258: H508-H514. 
30 Mori T, Polichnowski A, Glocka P, Kaldunski M, Ohsaki Y, Liang M, Cowley Jr AW. High perfusion pressure accelerates renal injury in salt-sensitive hypertension. J Am Soc Nephrol 2008; 19: 1472-1482.

31 Fukuo K, Yang J, Yasuda O, Mogi M, Suhara T, Sato N, Suzuki T, Morimoto S, Ogihara T. Nifedipine indirectly upregulates superoxide dismutase expression in endothelial cells via vascular smooth muscle cell-dependent pathways. Circulation 2002; 106: 356-361.

32 Matsui T, Takeuchi M, Yamagishi S. Nifedipine a calcium channel blocker, inhibits inflammatory and fibrogenic gene expressions in advanced glycation end product (AGE)-exposed fibroblasts via mineralocorticoid receptor antagonistic activity. Biochem Biophys Res Commun 2010; 396: 566-570.
33 Pergola PE, Raskin P, Toto RD, Meyer CJ, Huff JW, Grossman EB, Krauth M, Ruiz S, Audhya P, Christ-Schmidt H, Wittes J, Warnock DG. Bardoxolone methyl and kidney function in CKD with type 2 diabetes. N Engl J Med 2011; 365: 327-336.

(c) This work is licensed under the Creative Commons

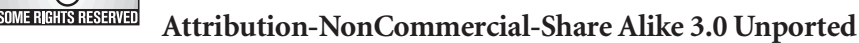
License. To view a copy of this license, visit http://creativecommons. org/licenses/by-nc-sa/3.0/ 